\title{
Designing polymers for nuclear track detection
}

\author{
V S Nadkarni \\ Department of Chemistry, Goa University, Goa-403 206, India \\ E-mail : nitin@unigoa.ac.in
}

\begin{abstract}
This article describes our attempts since 1996 to systematically design and synthesize monomers and corresponding polymers for nuclear track detection. So far more than 15 homopolymers/copolymers have been prepared and tested by us for this purpose. Some of these polymers show better track detection characteristics vis-á-vis commercially available poly allyl diglycol carbonate (PADC) track detectors.
\end{abstract}

Keywords : Nuclear tracks, homopolymer, copolymers, charged particle, polymeric track detectors, radiation detection.

PACS Nos. : $29.40 . \mathrm{Wk}, 81.05 . \mathrm{Lg}, 81.05 . \mathrm{Zx}, 81.16 . \mathrm{Be}, 82.35 .-\mathrm{x}, 82.75 . \mathrm{Fq}$

\section{Introduction}

Chemical Abstracts survey of literature pertaining to plastic/polymeric Solid State Nuclear Track Detectors (SSNTDs) shows that there are about 4300 research papers/ conference articles etc. that have been published by track workers during the period 1964-2006. A survey for the period 1964-1994 indicated that out of approx. 2000 articles on polymeric track detectors only about 2-3\% deals with design/preparation of polymeric detector films. Most of these $2-3 \%$ articles were devoted to preparation of PADC or Cellulose Nitrate (CN) detectors. During 1995-2006 the number of articles pertaining to design/preparation of polymeric track detectors was further reduced although another approximately 2300 research articles were published.

What is worth noting by all SSNTD workers is that there have not been any special attempts worldwide to design/prepare newer polymeric track detectors. With few exceptions, track workers have directed their attention either towards PADC (e.g. CR-39) or testing any readily available polymer for track detection. As a reason, therefore, no better polymeric materials than PADC, are commercially available to track workers.

Since 1996 our group at Goa University is engaged in designing and preparing 
new monomers/polymers for nuclear track detection. Considering the radiation sensitivity of various groups like $-\mathrm{ONO}_{2}$ (nitrate ester), - $\mathrm{CNO}_{2}$, (nitro), -OCOO- (carbonate), -NCOO(carbamate), -O-SO-O- (sulphite), -O- $\mathrm{SO}_{2}$ - (sulphonate) we have designed various allylic monomers that give thermoset, cross linked polymer matrix with different density of crosslinks. During these studies, we developed nine monomers including Allyl Diglycol Carbonate (ADC) and Diethylene Glycol bis(allyl sulfonate) (DEAS); six of these being novel. We also prepared their homopolymers and copolymers with ADC monomer. In the past six years we have indigenously developed and successfully tested about 15 new polymeric materials for track detection. It is heartening to note that one can have much better polymers than PADC for track applications.

\section{Materials and methods}

Most of the monomers were synthesized using the conventional transesterification method or by condensing chloroformates or the chlorosulphonates with glycol/alcohol. The $-\mathrm{ONO}_{2}$ group was introduced by nitration of corresponding alcohol. The monomers containing even more than one radiation sensitive groups with functionality ranging from two to eight were synthesized in this way. All the unbranched monomers [1-5] containing two identical radiation sensitive group like carbonate, sulphonate or sulphite have a functionality of four and were synthesized as shown in Scheme 1.

(1)

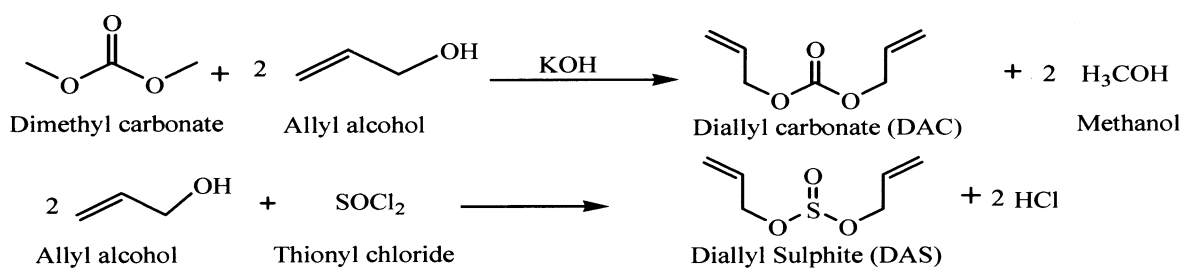

(3)

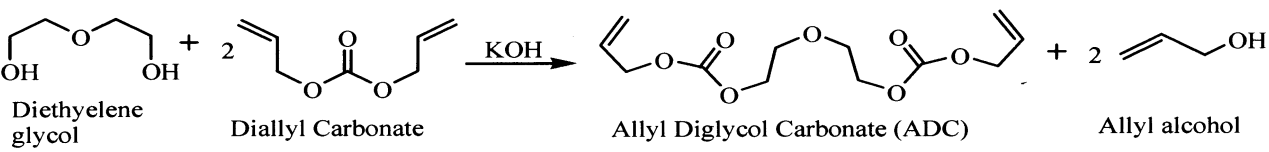

(4)

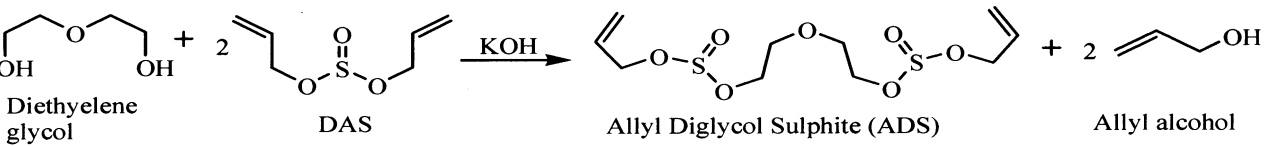

(5)
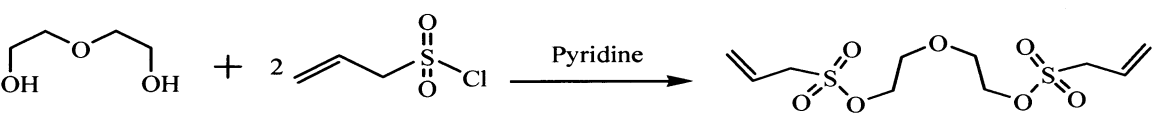

Diethylene glycol

Allyl chloro sulphonate

Diethylene glycol bis(allylsulphonate) (DEAS)

Scheme 1. Synthetic routes to some unbranched monomers.

Branched monomers [6-9] with higher functionality ranging from two to eight and containing more than two radiation sensitive groups like nitrate, nitro, carbamate, carbonate were used as shown in Schem 2, to prepare polymers so that more and more dense, 3D network of cross links is formed. 
(1)

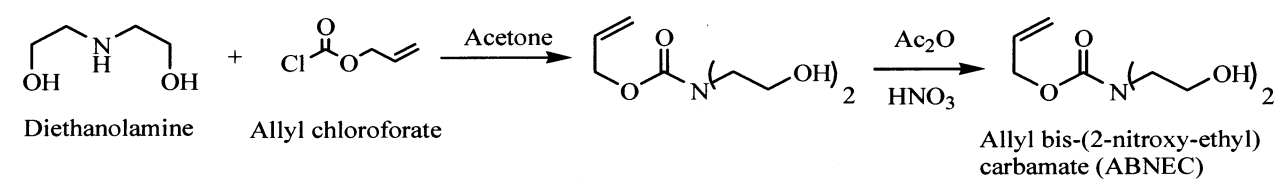

(2)

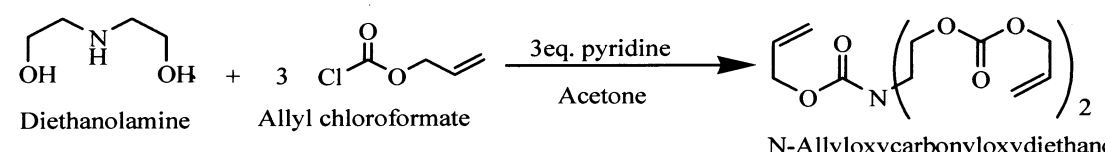
N-Allyloxycarbonyloxydiethanolaminebis (allyl carbonate)(NADAC)

(3)

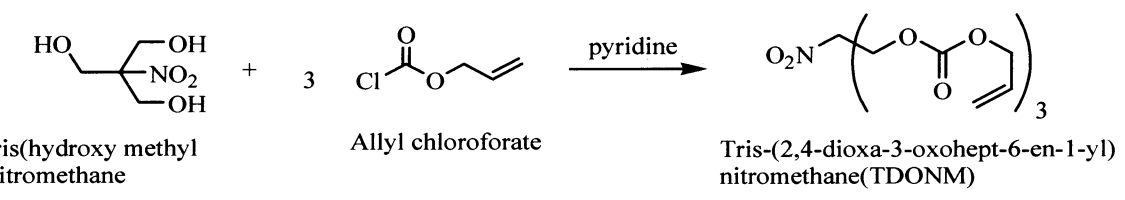

(4)
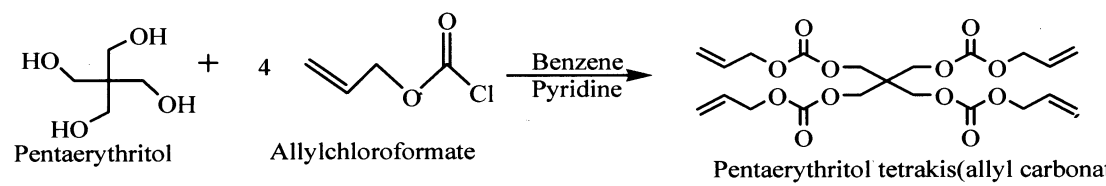

Pentaerythritol tetrakis(allyl carbonate) (PETAC)

Scheme 2. Synthetic routes to some branched monomers.

Table 1 summarizes some important characteristics of these monomers from polymerization point of view.

Table 1. Structural feature of the monomers.

\begin{tabular}{|c|c|c|c|c|c|}
\hline $\begin{array}{l}\text { Sl. } \\
\text { No. }\end{array}$ & $\begin{array}{c}\text { Monomer } \\
\text { name }\end{array}$ & $\begin{array}{c}\text { Functio- } \\
\text { nality }\end{array}$ & $\begin{array}{l}\text { Radiation } \\
\text { group }\end{array}$ & Sensitive & $\begin{array}{l}\text { Monomer } \\
\text { structure }\end{array}$ \\
\hline & & & Structure & Name & \\
\hline 1. & ADC & 04 & -O-CO-O- & carbonate & linear \\
\hline 2. & DAC & 04 & -O-CO-O- & carbonate & linear \\
\hline 3. & DAS & 04 & -O-SO-O- & sulphite & linear \\
\hline 4. & DEAS & 04 & $-\mathrm{O}-\mathrm{SO}_{2}-$ & sulfonate & linear \\
\hline 5. & PETAC & 08 & -O-CO-O- & carbonate & branched \\
\hline 6. & ADS & 04 & -O-SO-O- & sulphite & linear \\
\hline 7. & NADAC & 06 & $\begin{array}{l}-\mathrm{O}-\mathrm{CO}-\mathrm{O}-\text { and } \\
-\mathrm{N}-\mathrm{CO}-\mathrm{O}-\end{array}$ & $\begin{array}{c}\text { carbonate and } \\
\text { carbamate }\end{array}$ & branched \\
\hline 8. & ABNEC & 02 & $\begin{array}{c}-\mathrm{N}-\mathrm{CO}-\mathrm{O}-\text { and } \\
-\mathrm{O}-\mathrm{NO}_{2}\end{array}$ & $\begin{array}{c}\text { carbonate and } \\
\text { nitrate }\end{array}$ & branched \\
\hline 9. & TDONM & 06 & $\begin{array}{c}-\mathrm{O}-\mathrm{CO}-\mathrm{O}-\text { and } \\
-\mathrm{C}-\mathrm{NO}_{2}\end{array}$ & $\begin{array}{c}\text { carbonate and } \\
\text { nitro }\end{array}$ & branched \\
\hline
\end{tabular}

\subsection{Polymerization :}

500-600 micron films from these monomers were then prepared using a mold assembly designed to cast polymerize these monomers along with suitable initiator ( $3-6 \%$ by wt) 
and plasticizer like Di-isoOctyl Phthalate (DOP) (up to $1-2 \%$ by wt). A study of kinetics of allylic polymerization was also carried out in many cases and the kinetic model proposed by Dial et al was successfully extended to polymerization of some of the monomers like NADAC, TDONM, PETAC etc. This helped us in casting polymer films which are relatively free from defects like cracking or coloration.

\section{Results and discussion}

Monomers containing allyl $\left(\mathrm{CH}_{2}=\mathrm{CH}-\mathrm{CH}_{2}-\right)$ group were prepared as allyl group undergoes polymerization and can give a $3 \mathrm{D}$ polymer network if functionality of the monomer is more than two. If some functional groups containing hetero atoms $(\mathrm{N}, \mathrm{O}, \mathrm{S}, \mathrm{P})$ are introduced by suitable synthetic procedure between two allyl groups, these hetero atoms in the functional group become a source of delta rays as proposed earlier [10]. We have prepared monomers containing one of the functional groups like $-\mathrm{ONO}_{2}$, -O-CO-O-, -N-CO-O-, O-SO-O-, -O- $\mathrm{SO}_{2}$ - or a monomer with more than one type of functional group. Further to improve density of crosslink's in a 3D polymer chain network, we have used branched monomers (with one or two branches). We have also prepared some monomers which have pendant groups to see the effect on radiation sensitivity. Based on our experience over last 15 years, we have developed a protocol that may be useful for a track worker who wants to design and synthesize new monomers/polymers for track detection. This is briefly described below :

Step 1: Initial studies of a monomer/polymer:

(a) Designing of the monomer, syntheses and its spectral characterization and testing. Thermal stability of the monomer - Thermogravimetry/ Differential Thermal Analysis (TG/DTA).

(b) Casting a test polymer sample and its characterization w.r.t. color of the film, softness, \% unsaturation left, average thickness and spectral properties.

(c) Exposure to radiation source and etching in $6 \mathrm{~N} \mathrm{NaOH}$ at $70^{\circ} \mathrm{C}$.

(d) Determination of bulk etch rate $\left(V_{b}\right)$.

(e) If necessary, (depending upon post etch appearance and response of the film), change etchant concentration/temperature and etching time till tracks are observed. Observe whether a polymer reveals only fission tracks or alpha tracks as well.

$(\mathrm{f})$ Prepare copolymer with ADC (or other monomers) and test as above.

Step 2 : Kinetics of polymerization :

Apply Dial's kinetic model [11] to pure allylic monomer/to mixture of allylic monomers and generate a constant rate polymerization cycle. If monomer degrades thermally, restrict Dial's model up to that specific temperature. 
Step 3 : Optimization of initiator concentration :

(a) Prepare films using different concentrations of initiator/using different initiators.

(b) Expose to $\mathrm{Cf}^{252}$ at 4 or $5 \mathrm{~cm}$ from source in vacuum and etch at suitable conditions as observed above.

(c) Find the sensitivity by noting diameters of alpha/fission tracks.

(d) Repeat for all the films prepared with different initiator concentrations.

(e) Determine the optimum initiator concentration where sensitivity is maximum.

Step 4: Optimization of etching conditions :

(a) Prepare enough films at optimized initiator concentration and expose to fission/alpha source.

(b) Etch the films, keeping temperature of etchant constant but varying normality/keeping normality constant but varying temperature.

(c) Note $V_{b}$ at every etching interval and also track appearance time; Select those conditions (normality and temperature) at which $V_{b}$ is moderate so that track development occurs within a reasonable time, post etch appearance of the film is good.

Step 5 : Determination of alpha sensitivity (S) at optimized etching conditions :

At the selected optimum conditions, expose freshly prepared films to $\mathrm{Cf}^{252}$ in vacuum, etch and observe the variation of $V_{b}$ and sensitivity as a function of time to determine optimum etching time where $S$ is maximum. Use the optimized etching conditions obtained above.

Step 6 : Other studies :

(1) Determination of threshold energy/alpha/ff branching ratio.

(2) For the films prepared above at different initiator concentrations, record TG/DTA as well as XRD patterns.

(3) Compare the $V_{b} /$ track detection efficiency as a function of glass transition, XRD (amorphous/crystalline nature) i.e., in turn with initiator concentration.

(4) Study of these films for neutron/proton/gamma dosimetry.

(5) Electrochemical etching studies for all films.

We followed this particular protocol to maintain uniformity in our studies and got better polymer films that give more consistent/acceptable results. Table 2 gives a summary of track detection characteristics of the polymers prepared so far by us. A little higher background track like features due to matrix faults generated in the films during 
Table 2. Track detection characteristics of polymers prepared.

\begin{tabular}{|c|c|c|c|c|c|c|}
\hline \multirow[t]{2}{*}{$\begin{array}{l}\text { Sl. } \\
\text { No. }\end{array}$} & \multirow[t]{2}{*}{ Polymer composition } & \multirow[t]{2}{*}{$\begin{array}{l}\text { Particles } \\
\text { detected }\end{array}$} & \multirow[t]{2}{*}{$\begin{array}{l}\text { Alpha } \\
\text { sensitivity }\end{array}$} & \multirow[t]{2}{*}{ Etching conditions } & \multicolumn{2}{|c|}{$\begin{array}{l}\text { Track development } \\
\text { Time (min) }\end{array}$} \\
\hline & & & & & Alpha & Fission \\
\hline 1. & ADC homopolymer & $\alpha$ and fission & $1.3-1.35$ & $6 \mathrm{~N} \mathrm{NaOH}, 70^{\circ} \mathrm{C}$ & - & - \\
\hline 2. & NADAC homopolymer & $\alpha$ and fission & 1.35 & $6 \mathrm{~N} \mathrm{NaOH}, 80^{\circ} \mathrm{C}$ & 480 & 60 \\
\hline 3. & NADAC : ADC $(1: 1)$ & $\alpha$ and fission & 1.7 & $6 \mathrm{~N} \mathrm{NaOH}, 75^{\circ} \mathrm{C}$ & 360 & 60 \\
\hline 4. & ABNEC : ADC $(1: 1)$ & fission & - & $6 \mathrm{~N} \mathrm{NaOH}, 70^{\circ} \mathrm{C}$ & - & 20 \\
\hline 5. & ABNEC : ADC $(1: 9)$ & $\alpha$ and fission & 1.13 & $6 \mathrm{~N} \mathrm{NaOH}, 70^{\circ} \mathrm{C}$ & 420 & 45 \\
\hline 6. & DAS : ADC (2:8) & $\alpha$ and fission & $(\alpha$ at $3 \mathrm{~cm})$ & $6 \mathrm{~N} \mathrm{NaOH}, 70^{\circ} \mathrm{C}$ & 20 & 10 \\
\hline 7. & DAC homopolymer & fission & & $6 \mathrm{~N} \mathrm{NaOH}, 70^{\circ} \mathrm{C}$ & - & 60 \\
\hline 8. & DAC : ADC $(1: 1)$ & $\alpha$ and fission & & $6 \mathrm{~N} \mathrm{NaOH}, 80^{\circ} \mathrm{C}$ & 240 & 60 \\
\hline 9. & DEAS : ADC (3: 7) & $\alpha$ and fission & 2.38 & $6 \mathrm{~N} \mathrm{NaOH}, 70^{\circ} \mathrm{C}$ & 60 & 30 \\
\hline 10. & DEAS : ADC $(2: 8)$ & $\alpha$ and fission & 1.39 & $6 \mathrm{~N} \mathrm{NaOH}, 70^{\circ} \mathrm{C}$ & 60 & 30 \\
\hline 11. & DEAS homopolymer & $\alpha$ and fission & hazy film & $6 \mathrm{~N} \mathrm{NaOH}, 70^{\circ} \mathrm{C}$ & 180 & 60 \\
\hline 12. & PETAC homopolymer & $\alpha$ and fission & 1.33 & $6 \mathrm{~N} \mathrm{NaOH}, 70^{\circ} \mathrm{C}$ & 120 & 45 \\
\hline 13. & PETAC : ADC $(1: 1)$ & $\alpha$ and fission & 1.45 & $6 \mathrm{~N} \mathrm{NaOH}, 70^{\circ} \mathrm{C}$ & 90 & 45 \\
\hline 14. & ADS : ADC $(1: 9)$ & $\alpha$ and fission & hazy film & $6 \mathrm{~N} \mathrm{NaOH}, 70^{\circ} \mathrm{C}$ & 20 & 10 \\
\hline 15. & TDONM homopolymer & $\alpha$ and fission & $(\alpha$ at $3 \mathrm{~cm})$ & $4 \mathrm{~N} \mathrm{NaOH}, 60^{\circ} \mathrm{C}$ & 240 & 60 \\
\hline 16. & TDONM : ADC (2:8) & $\alpha$ and fission & 1.22 & $4 \mathrm{~N} \mathrm{NaOH}, 60^{\circ} \mathrm{C}$ & 240 & 60 \\
\hline 17. & TDONM : ADC (1:9) & $\alpha$ and fission & 1.45 & $4 \mathrm{~N} \mathrm{NaOH}, 60^{\circ} \mathrm{C}$ & 240 & 60 \\
\hline
\end{tabular}

polymerization, incorporated minute dust particles or gas bubbles etc., is a problem which one has to address, especially when films are required for low level measurements. This problem can be solved to a considerable extent by synthesizing the monomer/ polymer in a dust free environment, proper monomer filtration stages and taking special care while feeling the molds with monomer.

\section{Conclusion}

Based on our experience over past 15 years of studies in designing monomers/ polymers for track detection, we have generated a protocol to cast thin track detector films from allylic monomers and their testing for track detection. It is possible to prepare polymers having better track detection characteristics than commercially available films like CR-39.

\section{Acknowledgments}

The author wishes to thank the Atomic Energy Regulatory Board, Government of India, for providing research grants to him during the years 1998-2007. Thanks are also due to National Institute of Oceanography, Goa and Indian Institute of Science, Bangalore, India for providing NMR and MS spectral data respectively. The author also wishes to thank Prof. S D Samant, UICT, Mumbai; Dr. A M Bhagwat, Ex-Head, RSSD, BARC; 
Dr. S B Manohar, Ex-Head, RCD, BARC; Dr. Hari Moahn, Ex-Head, RCS, RCCD, BARC; Dr. V K Manchanda, Head, RCD, BARC, Dr. S Sabharwal, Head, RTDS, BARC and Dr. P C Kalsi, SO"G", RCD, BARC for their help and encouragement from time to time. The author also wishes to thank Dr. S G Tilve, Reader, Department of Chemistry; Mr. Vinod and Mr. Adlete for their help in this work.

\section{References}

[1] V K Mandrekar, S G Tilve and V S Nadkarni National Symposium on Radiation and Photochemistry, Abstract RC-7 (2007)

[2] V K Mandrekar, S G Tilve and V S Nadkami Trombay Symposium on Radiation and Photochemistry, Abstracts px-55 (2006)

[3] V S Nadkami, S G Tilve and A A A Mascarenhas Indian Process Patent no. IP 196627 (1999)

[4] V K Mandrekar, S G Tilve and V S Nadkami 23rd Int. Conf. Nuclear Tracks in Solids, Abstracts px-219 (2006)

[5] A A A Mascarenhas, R V Kolekar, P C Kalsi, A Ramaswami, V B Joshi, S G Tilve and V S Nadkami Radiation Measurements 4122 (2006)

[6] M Fujii, R Yokota and Y Atarshi Nucl. Tracks Radiat. Meas. 1719 (1990)

[7] A A A Mascarenhas, S G Tilve and V S Nadkami Designed Monomers and Polymers 8177 (2005)

[8] A A A Mascarenhas, V K Mandrekar, P C Kalsi, S G Tilve and V S Nadkami 23rd Int. Conf. Nuclear Tracks in Solids, Abstracts px-218 (2006)

[9] V K Mandrekar, S G Tilve and V S Nadkami 23rd Int. Conf. Nuclear Tracks in Solids, Abstracts px-220 (2006)

[10] M Fujii and R Yokota Nucl. Tracks 1255 (1986)

[11] W R Dial, W E Bissinger, B J De Witt and F Strain Ind. Engg. Chem. 472447 (1955) 